\title{
Borcherds products associated with certain Thompson series
}

\author{
Chang Heon Kim
}

\begin{abstract}
We apply Zagier's result for the traces of singular moduli to construct Borcherds products in higher level cases.
\end{abstract}

\section{Introduction}

Let $M_{1 / 2}^{!}$be the additive group consisting of nearly holomorphic modular forms of weight $\frac{1}{2}$ for $\Gamma_{0}(4)$ whose Fourier coefficients are integers and satisfy the Kohnen's 'plus space' condition (i.e. $n$th coefficients vanish unless $n \equiv 0$ or 1 modulo 4 ). We also let $\mathcal{B}$ be the multiplicative group consisting of meromorphic modular forms for some characters of $S L_{2}(\mathbb{Z})$ of integral weight with leading coefficient 1 whose coefficients are integers and all of whose zeros and poles are either cusps or imaginary quadratic irrationals. Borcherds [Bor95] gave an isomorphism between $M_{1 / 2}^{!}$and $\mathcal{B}$ by means of infinite products which we call modular products or Borcherds products.

Let $d$ denote a positive integer congruent to 0 or 3 modulo 4 . We denote by $\mathcal{Q}_{d}$ the set of positive definite binary quadratic forms $Q=[a, b, c]=a X^{2}+b X Y+c Y^{2}(a, b, c \in \mathbb{Z})$ of discriminant $-d$, with usual action of the modular group $\Gamma=P S L_{2}(\mathbb{Z})$. To each $Q \in \mathcal{Q}_{d}$, we associate its unique root $\alpha_{Q} \in \mathfrak{H}$ (= upper half plane). We define the Hurwitz-Kronecker class number $H(d)$ by $H(d)=\sum_{Q \in \mathcal{Q}_{d} / \Gamma}\left(1 / w_{Q}\right)$ where $w_{Q}=\left|\Gamma_{Q}\right|$. For instance, we have $H(3)=\frac{1}{3}, H(4)=\frac{1}{2}$, $H(7)=H(8)=H(11)=1, H(12)=\frac{4}{3}, H(15)=2$, etc. For the modular invariant $j(\tau)$, we define a function $\mathcal{H}_{d}(j(\tau)) \in \mathcal{B}$ by $\prod_{Q \in \mathcal{Q}_{d} / \Gamma}\left(j(\tau)-j\left(\alpha_{Q}\right)\right)^{1 / w_{Q}}$. On the other hand, for each $d$ there is a unique modular form $f_{d, 1} \in M_{1 / 2}^{!}$having a Fourier development of the form $f_{d, 1}=$ $q^{-d}+\sum_{D>0} A(D, d) q^{D}, q=e^{2 \pi i \tau}(\tau \in \mathfrak{H})$. Then Borcherds' theorem states that

$$
\mathcal{H}_{d}(j(\tau))=q^{-H(d)} \prod_{u=1}^{\infty}\left(1-q^{u}\right)^{A\left(u^{2}, d\right)} .
$$

Zagier [Zag00] described the trace of a singular modulus of discriminant $-d\left(=\sum_{Q \in \mathcal{Q}_{d} / \Gamma}\left(1 / w_{Q}\right)\right.$ $\left.\left(j\left(\alpha_{Q}\right)-744\right)\right)$ as the coefficient of $q^{d}$ in a fixed modular form $-g_{1,1}(\tau)$ of weight $\frac{3}{2}$. By making use of this formula and considering Hecke operators in integral and half-integral weight, Zagier reproved $(*)$ (see $[\mathrm{Zag} 00, \S 6]$ ). Moreover he generalized the trace formula to the group $\Gamma_{0}(N)^{*}$ (which is the group generated by $\Gamma_{0}(N)$ and all Atkin-Lehner involutions $W_{e}$ for $\left.e \| N\right)$ for $2 \leqslant N \leqslant 6$ (see [Zag00, $\left.\S 8\right]$ ).

In this article we find an analogue of $(*)$ in higher level cases $N=2,3,5,6$ by applying Zagier's Theorem 8 of [Zag00]. Let $M_{k-1 / 2}^{+\cdots+}(N)^{!}$be the vector space consisting of nearly holomorphic modular forms of half-integral weight $k-\frac{1}{2}$ on $\Gamma_{0}(4 N)$ whose $n$th Fourier coefficient vanishes unless $(-1)^{k-1} n$ is a square modulo $4 N$. There is a unique modular form $f_{d, N} \in M_{1 / 2}^{+\cdots+}(N)$ ! having a Fourier

Received 13 February 2002, accepted in final form 5 June 2002.

2000 Mathematics Subject Classification 11F03, 11F11, 11F22, $11 \mathrm{~F} 50$.

Keywords: modular product, generalized Hecke operator, Jacobi form, half integral form.

This journal is (C) Foundation Compositio Mathematica 2004. 


\section{H. KIM}

expansion of the form

$$
f_{d, N}=q^{-d}+\sum_{D>0} A(D, d) q^{D}
$$

An explicit construction of $f_{d, N}$ is given in Appendix A and the uniqueness of $f_{d, N}$ is shown in the end of $\S 2$. Let $\mathcal{Q}_{d, N}$ be the set of forms $Q=[a, b, c] \in \mathcal{Q}_{d}$ satisfying $N \mid a$. Then $\Gamma_{0}(N)^{*}$ naturally acts on $\mathcal{Q}_{d, N}$ and the quotient $\mathcal{Q}_{d, N} / \Gamma_{0}(N)^{*}$ has a bijection with $\mathcal{Q}_{d} / \Gamma$ (see [Zag00, $\left.\S 8\right]$ ). We can therefore define, for the Hauptmodul $t(\tau)$ for $\Gamma_{0}(N)^{*}$, a modular function $\mathcal{H}_{d}(t(\tau))$ by $\prod_{Q \in \mathcal{Q}_{d, N} / \Gamma_{0}(N)^{*}}\left(t(\tau)-t\left(\alpha_{Q}\right)\right)^{1 / w_{Q}}$. In $\S 3$ we prove the following theorem.

Theorem 1.1. Let $1 \leqslant N \leqslant 6$ other than 4 and $t$ be the Hauptmodul for $\Gamma_{0}(N)^{*}$. Let $-d$ be the discriminant corresponding to a Heegner point (i.e. the discriminant of $Q \in \mathcal{Q}_{d, N}$ with the condition that if $f^{2}$ divides $d$, then $\left.(f, N)=1\right)$. Define $A^{*}\left(u^{2}, d\right)=2^{s(u, N)} A\left(u^{2}, d\right)$ where $s(u, N)$ is the number of distinct prime factors dividing $(u, N)$. Then

$$
\mathcal{H}_{d}(t(\tau))=q^{-H(d)} \prod_{u=1}^{\infty}\left(1-q^{u}\right)^{A^{*}\left(u^{2}, d\right)} .
$$

We remark that this theorem is related to the problem of generalizing Borcherds' theorem [Bor95, Theorem 14.1] to higher levels [Bor95, problem 10, § 17]. In some sense Borcherds proved it himself [Bor98, Theorem 13.3]. The vector valued modular forms he uses include the higher level case, because a higher level form can be induced up to a vector valued form of level 1. An explicit infinite product is given in part 5 of Theorem 13.3 of [Bor98]. However, as he pointed out, it seems to take a bit of effort to unravel it to see what it says in the case of modular forms. Also, Bruinier [Bru02] proved that every automorphic form with zeros on Heegner divisors can be written as modular products in the case that the lattice considered splits two hyperbolic planes over $\mathbb{Z}$.

Finally, in $\S 4$, by using the idea given in [KKKO], we derive a recursion formula which enables us to estimate all $A^{*}\left(u^{2}, d\right)$ for $u \geqslant 1$ from the Fourier coefficients of $\mathcal{H}_{d}(t(\tau))$.

\section{Preliminaries}

\subsection{Generalized Hecke operator}

Let $N$ be a positive integer and $e$ be any Hall divisor of $N$ (written $e \| N$ ), that is, a positive divisor of $N$ for which $(e, N / e)=1$. We denote by $W_{e, N}$ a matrix $\left(\begin{array}{cc}a e & b \\ c N & d e\end{array}\right)$ with $\operatorname{det} W_{e, N}=e$ and $a, b, c, d \in \mathbb{Z}$, and call it an Atkin-Lehner involution. Let $S$ be a subset of Hall divisors of $N$ and let $N+S$ be the subgroup of $P S L_{2}(\mathbb{R})$ generated by $\Gamma_{0}(N)$ and all Atkin-Lehner involutions $W_{e, N}$ for $e \in S$ (we may choose $S$ so that $1 \notin S$ and if $e_{1}, e_{2} \in S$, then $e_{1} e_{2} /\left(e_{1}, e_{2}\right)^{2} \in S$ unless $\left.e_{1}=e_{2}\right)$. We assume that the genus of the group $N+S$ is zero. Then there exists a unique modular function $t$ with respect to $N+S$ satisfying:

i) $t$ is holomorphic on the complex upper half plane $\mathfrak{H}$;

ii) $t$ has the Fourier expansion at $\infty$ of the form

$$
t=q^{-1}+\sum_{k \geqslant 1} H_{k} q^{k}, \quad q=e^{2 \pi i \tau}(\tau \in \mathfrak{H}) ;
$$

iii) $t$ is holomorphic at all cusps which are not equivalent to $\infty$ under $N+S$.

Such a function $t$ is called the Hauptmodul for $N+S$. By the result of Borcherds [Bor92], $t$ becomes a monstrous function whose Fourier coefficients are related to representations of the monster group $\mathbb{M}$ except for three cases $(25-, 49+49$ and $50+50)$. More precisely the $q$-series of $t$ coincides with a Thompson series $T_{g}(\tau)=\sum_{n \in \mathbb{Z}} \operatorname{Tr}\left(g \mid V_{n}\right) q^{n}$ for some element $g$ of $\mathbb{M}$. Here $V=\bigoplus_{n \in \mathbb{Z}} V_{n}$ is the infinite-dimensional graded representation of $\mathbb{M}$ constructed by Frenkel et al. [FLM84, FLM88]. 


\section{BORCHERds PRODUCTS ASSOCIATED WITH CERTAIN THOMPSON SERIES}

For a prime number $p$, let $t^{(p)}$ be the Hauptmodul for $N^{(p)}+S^{(p)}$ where $N^{(p)}=N /(p, N)$ and $S^{(p)}$ is the set of all $e$ in $S$ which divide $N^{(p)}$. In general, if $m=p_{1} p_{2} \cdots p_{r}$ is a product of primes $p_{i}$, then we define the $m$ th replicate $t^{(m)}$ of $t$ by

$$
t^{(m)}=\left(\cdots\left(\left(t^{\left(p_{1}\right)}\right)^{\left(p_{2}\right)} \cdots\right)^{\left(p_{r}\right)} .\right.
$$

For every positive integer $n$, let $t_{n}$ be a unique polynomial of $t$ satisfying $t_{n} \equiv q^{-n} \bmod q \mathbb{C}[[q]]$. Define the $m$ th generalized Hecke operator $T(m)$ [ACMS92, Fer96a, Fer96b, Koi] by

$$
\left.t_{n}\right|_{T(m)}=\sum_{\substack{a d=m \\ 0 \leqslant b<d}} t_{n}^{(a)}\left(\frac{a \tau+b}{d}\right) .
$$

The $m$ th replication formula [Fer96b, Koi] states that $t_{m}=\left.t\right|_{T(m)}$.

\subsection{Jacobi forms}

A (holomorphic) Jacobi form on $S L_{2}(\mathbb{Z})$ is defined to be a holomorphic function $\phi: \mathfrak{H} \times \mathbb{C} \rightarrow \mathbb{C}$ satisfying the two transformation equations

$$
\begin{gathered}
\phi\left(\frac{a \tau+b}{c \tau+d}, \frac{z}{c \tau+d}\right)=(c \tau+d)^{k} e^{2 \pi i N c z^{2} /(c \tau+d)} \phi(\tau, z) \quad\left(\left(\begin{array}{ll}
a & b \\
c & d
\end{array}\right) \in S L_{2}(\mathbb{Z})\right), \\
\phi(\tau, z+\lambda \tau+\mu)=e^{-2 \pi i N\left(\lambda^{2} \tau+2 \lambda z\right)} \phi(\tau, z) \quad\left((\lambda \mu) \in \mathbb{Z}^{2}\right)
\end{gathered}
$$

and having a Fourier expansion of the form

$$
\phi(\tau, z)=\sum_{\substack{n, r \in \mathbb{Z} \\ 4 N n-r^{2} \geqslant 0}} c(n, r) q^{n} \zeta^{r} \quad\left(q=e^{2 \pi i \tau}, \zeta=e^{2 \pi i z}\right) .
$$

Here $k$ and $N$ are positive integers called the weight and index of $\phi$, respectively. The coefficient $c(n, r)$ depends only on $4 N n-r^{2}$ and on $r(\bmod 2 N)$ [EZ85, Theorem 2.2]. In (1), if the condition $4 N n-r^{2} \geqslant 0$ is deleted, we obtain a nearly holomorphic Jacobi form.

Let $J_{k, N}^{!}$be the space of nearly holomorphic Jacobi forms of weight $k$ and index $N$. Let $J_{*, *}^{!}$ be the ring of all nearly holomorphic Jacobi forms and $J_{\mathrm{ev}, *}^{!}$its even weight subring. Then $J_{\mathrm{ev}, *}^{!}$is the free polynomial algebra over $M_{*}^{!}(\Gamma)=\mathbb{C}\left[E_{4}, E_{6}, \Delta^{-1}\right] /\left(E_{4}^{3}-E_{6}^{2}=1728 \Delta\right)$ on two generators $a=\tilde{\phi}_{-2,1}(\tau, z) \in J_{-2,1}^{!}$and $b=\tilde{\phi}_{0,1}(\tau, z) \in J_{0,1}^{!}$(for details, see [EZ85, $\left.\S 9\right]$ ). Fix $k=2$ and $1 \leqslant N \leqslant 6, \neq 4$. There are unique Jacobi forms $\phi_{D, N} \in J_{2, N}^{!}$having Fourier coefficients $c(n, r)=$ $B\left(D, 4 N n-r^{2}\right)$ which depend only on the discriminant $r^{2}-4 N n$ with $B(D,-D)=1$ and $B(D, d)=0$ if $d=4 N n-r^{2}<0, \neq-D$. The uniqueness of $\phi_{D, N}$ is obvious since the difference of any two functions satisfying the definition of $\phi_{D, N}$ would be an element of $J_{2, N}$ (the space of holomorphic Jacobi forms of weight 2 and index $N$ ), which is of dimension zero by [EZ85, Theorem 9.1(2)]. For the existence, we need the additional condition on Fourier coefficients that

$$
B(D, 0)= \begin{cases}-2, & \text { if } D \text { is a square } \\ 0, & \text { otherwise. }\end{cases}
$$

The structure theorem then allows us to express $\phi_{D, N}$ as a linear combination of $a^{i} b^{N-i}(i=$ $0, \ldots, N)$ over $M_{*}^{!}(\Gamma)$. Define

$$
g_{D, N}=q^{-D}+\sum_{d \geqslant 0} B(D, d) q^{d} .
$$

By the correspondence between Jacobi forms and half-integral forms [EZ85, Theorem 5.6], $g_{D, N}$ lies in the space $M_{3 / 2}^{+\cdots+}(N)$ ! so that $f_{d, N} g_{D, N}$ defines a modular form of weight 2 for $\Gamma_{0}(4 N)$. We write $f_{d, N} g_{D, N}=\sum_{n \in \mathbb{Z}} c_{n} q^{n}$. The 'plus' conditions imposed on $f_{d, N}$ and $g_{D, N}$ force $\left.\left(f_{d, N} g_{D, N}\right)\right|_{U_{4 N}}$ 


\section{H. KIM}

to be a modular form of weight 2 on $S L_{2}(\mathbb{Z})$. Here $U_{4 N}$ is the operator sending $\sum_{n \in \mathbb{Z}} c_{n} q^{n}$ to $\sum_{n \in \mathbb{Z}} c_{4 N n} q^{n}$. In fact, if we consider

$$
h=\sum_{i \in(\mathbb{Z} / 4 N \mathbb{Z})^{\times}}\left(f_{d, N} g_{D, N}\right)\left(\frac{\tau+i}{4 N}\right),
$$

then $h$ is invariant under the action of $\left(\begin{array}{cc}0 & -1 \\ 1 & 0\end{array}\right)$ and has the Fourier development of the form $\varphi(4 N) \sum_{n \in \mathbb{Z}} e^{2 \pi i n / N} c_{4 n} q^{n / N}$ since $c_{n}$ vanishes whenever $n \equiv 2 \bmod 4 . \sum_{i=0}^{N-1} h(\tau+i)=N \varphi(4 N)$ $\left.\left(f_{d, N} g_{D, N}\right)\right|_{U_{4 N}}$ is then invariant under the action of $S L_{2}(\mathbb{Z})$ with a pole only at $\infty$. Thus $\left(f_{d, N} g_{D, N}\right)$ $\left.\right|_{U_{4 N}}$ can be written as the derivative of some polynomial in $j$. By comparing the constant terms we get $A(D, d)=-B(D, d)$. This also shows the uniqueness of $f_{d, N}$.

Throughout the article we adopt the following notations.

Notation. $T(m)$ for the generalized Hecke operator; $T_{m}$ for the Hecke operator acting on Jacobi forms or half-integral forms [EZ85, $\S \S 4$ and 5]; $\phi_{D}=\phi_{D, N} ; g_{D}=g_{D, N} ; f_{d}=f_{d, N} ; \phi_{D}^{(p)}=\phi_{D, N^{(p)}}$; $g_{D}^{(p)}=g_{D, N(p)} ;$ and $B(d)=B(1, d)$.

\section{Proof of Theorem 1.1}

For each positive integer $m$ and prime $p$, we define

$$
J_{m}(d)=\sum_{Q \in \mathcal{Q}_{d, N} / \Gamma_{0}(N)^{*}} \frac{1}{w_{Q}} t_{m}\left(\alpha_{Q}\right)
$$

and



First we need two lemmas.

Lemma 3.1. Let $p$ be a prime dividing $N$. For $i \geqslant 0$ and $m$ coprime to $p$,

$$
\left.\phi_{p^{2 i} m^{2}}^{(p)}\right|_{V_{p}}=p \phi_{p^{2 i+2} m^{2}}+\phi_{p^{2 i} m^{2}} .
$$

Here $V_{p}$ is the Hecke operator on Jacobi forms defined by the formula (2) in [EZ85, § 4].

Proof. According to [EZ85, Theorem 4.1], the operator $V_{p}$ maps $J_{2, N / p}^{!}$to $J_{2, N}^{!}$. From the formula (7) in [EZ85, p. 43], we find that

$$
\text { the coefficient of } q^{n} \zeta^{r} \text { in }\left.\phi_{p^{2 i} m^{2}}^{(p)}\right|_{V_{p}}= \begin{cases}p, & \text { if } 4 N n-r^{2}=-p^{2 i+2} m^{2} \\ 1, & \text { if } 4 N n-r^{2}=-p^{2 i} m^{2} \\ 0, & \text { if } 4 N n-r^{2}<0, \neq-p^{2 i+2} m^{2},-p^{2 i} m^{2} .\end{cases}
$$

From these observations and the uniqueness of $\phi_{D}$, the lemma immediately follows.

Lemma 3.2. Let $l$ be a positive integer coprime to $N$ and $d=4 N n-r^{2}$. Then:

i) $J_{l}(d)=-$ coefficient of $q^{n} \zeta^{r}$ in $\left.\phi_{1}\right|_{T_{l}}$;

ii) $\left.\phi_{1}\right|_{T_{l}}=\sum_{\nu \mid l} \nu \phi_{\nu^{2}}$.

Proof. i) Let $p$ be a prime divisor of $l$. Then

$$
\begin{aligned}
J_{p}(d) & =\sum_{Q \in \mathcal{Q}_{d, N} / \Gamma_{0}(N)^{*}} \frac{1}{w_{Q}} t_{p}\left(\alpha_{Q}\right)=\left.\left.\sum_{Q \in \mathcal{Q}_{d, N} / \Gamma_{0}(N)^{*}} \frac{1}{w_{Q}} t\right|_{T(p)}(\tau)\right|_{\tau=\alpha_{Q}} \\
& =J_{1}\left(d p^{2}\right)+\left(\frac{-d}{p}\right) J_{1}(d)+p J_{1}\left(\frac{d}{p^{2}}\right)
\end{aligned}
$$

by a similar argument as that given in the proof of [Zag00, Theorem 5(ii)] 


\section{BORCHERds PRODUCTS ASSOCIATED WITH CERTAIN THOMPSON SERIES}

$$
\begin{aligned}
& =-\left[B\left(d p^{2}\right)+\left(\frac{-d}{p}\right) B(d)+p B\left(\frac{d}{p^{2}}\right)\right] \quad \text { by [Zag00, Theorem 8] } \\
& =-B_{p}(d) .
\end{aligned}
$$

Here $J_{1}\left(d / p^{2}\right)$ (respectively $B\left(d / p^{2}\right)$ ) is defined to be zero unless $d / p^{2}$ is an integer and $B_{p}(d)$ denotes the coefficient of $q^{d}$ in $\left.g_{1}\right|_{T_{p}}$, which is the same as the coefficient of $q^{n} \zeta^{r}$ in $\left.\phi_{1}\right|_{T_{p}}$ (see [EZ85, Theorems 4.5 and 5.4]). Now let $p^{s}|| l$. Observe that $\left.t\right|_{T\left(p^{s}\right)}=\left.t_{p^{s-1}}\right|_{T(p)}-p t_{p^{s-2}}(\tau)$. Thus

$$
\begin{aligned}
J_{p^{s}}(d) & =J_{p^{s-1}}\left(d p^{2}\right)+\left(\frac{-d}{p}\right) J_{p^{s-1}}(d)+p J_{p^{s-1}}\left(\frac{d}{p^{2}}\right)-p J_{p^{s-2}}(d) \\
& =-\left[B_{p^{s-1}}\left(d p^{2}\right)+\left(\frac{-d}{p}\right) B_{p^{s-1}}(d)+p B_{p^{s-1}}\left(\frac{d}{p^{2}}\right)\right]+p B_{p^{s-2}}(d) \quad \text { by induction on } s \\
& =- \text { coefficient of } q^{n} \zeta^{r} \text { in }\left[\left.\left(\left.\phi_{1}\right|_{T_{p^{s-1}}}\right)\right|_{T_{p}}-\left.p \phi_{1}\right|_{T_{p^{s-2}}}\right] \\
& =- \text { coefficient of } q^{n} \zeta^{r} \text { in }\left.\phi_{1}\right|_{T_{p^{s}}} \text { by }[\text { EZ85, Corollary 1, p. 51]. }
\end{aligned}
$$

Now write $l=l^{\prime} p^{s}$ with $\left(l^{\prime}, p\right)=1$. Let $n(l)$ be the number of prime factors of $l$. We will use induction on $n(l)$. If $n(l)=1$, it returns to the previous case. Now $\left.t\right|_{T(l)}=\left.t\right|_{T\left(l^{\prime}\right) T\left(p^{s}\right)}=\left.t_{l^{\prime}}\right|_{T\left(p^{s}\right)}=$ $\left.t_{l^{\prime} p^{s-1}}\right|_{T(p)}-p t_{l^{\prime} p^{s-2}}$ which yields that

$$
\begin{aligned}
J_{l}(d) & =J_{l^{\prime} p^{s-1}}\left(d p^{2}\right)+\left(\frac{-d}{p}\right) J_{l^{\prime} p^{s-1}}(d)+p J_{l^{\prime} p^{s-1}}\left(\frac{d}{p^{2}}\right)-p J_{l^{\prime} p^{s-2}}(d) \\
& =- \text { coefficient of } q^{n} \zeta^{r} \text { in }\left.\phi_{1}\right|_{T_{l}} \text { by induction on } s .
\end{aligned}
$$

ii) As before, let $p$ be a prime dividing $l$ and $p^{s}|| l$. First we show that $\left.\phi_{1}\right|_{T_{p^{s}}}=\sum_{i=0}^{s} p^{i} \phi_{p^{2 i}}$. Let $s=1$. Then the coefficient of $q^{d}$ in $\left.g_{1}\right|_{T_{p}}$ is

$$
B\left(d p^{2}\right)+\left(\frac{-d}{p}\right) B(d)+p B\left(\frac{d}{p^{2}}\right)= \begin{cases}1, & \text { if } d=-1 \\ p, & \text { if } d=-p^{2} \\ 0, & \text { if } d<0, \neq-1,-p^{2} .\end{cases}
$$

This implies $\left.g_{1}\right|_{T_{p}}=p g_{p^{2}}+g_{1}$ and therefore $\left.\phi_{1}\right|_{T_{p}}=p \phi_{p^{2}}+\phi_{1}$. Now let $s \geqslant 2$. Then

$$
\begin{aligned}
\left.\phi_{1}\right|_{T_{p^{s}}} & =\left.\left(\phi_{1} \mid T_{p^{s-1}}\right)\right|_{T_{p}}-\left.p \phi_{1}\right|_{T_{p^{s-2}}} \\
& =\left.\left(\sum_{i=0}^{s-1} p^{i} \phi_{p^{2 i}}\right)\right|_{T_{p}}-p \sum_{i=0}^{s-2} p^{i} \phi_{p^{2 i}} \quad \text { by induction on } s .
\end{aligned}
$$

For $i>0$, the coefficient of $q^{d}$ in $\left.g_{p^{2 i}}\right|_{T_{p}}$ is

$$
B\left(p^{2 i}, d p^{2}\right)+\left(\frac{-d}{p}\right) B\left(p^{2 i}, d\right)+p B\left(p^{2 i}, \frac{d}{p^{2}}\right)= \begin{cases}1, & \text { if } d=-p^{2 i-2} \\ p, & \text { if } d=-p^{2 i+2} \\ 0, & \text { if } d<0, \neq-p^{2 i-2},-p^{2 i+2} .\end{cases}
$$

This shows that

Thus

$$
\left.\phi_{p^{2 i}}\right|_{T_{p}}= \begin{cases}\phi_{p^{2 i-2}}+p \phi_{p^{2 i+2}}, & \text { if } i>0 \\ \phi_{1}+p \phi_{p^{2}}, & \text { if } i=0\end{cases}
$$

$$
\begin{aligned}
\left.\phi_{1}\right|_{p_{p^{s}}} & =\left.\left(\sum_{i=0}^{s-1} p^{i} \phi_{p^{2 i}}\right)\right|_{T_{p}}-p \sum_{i=0}^{s-2} p^{i} \phi_{p^{2 i}}=\sum_{i=1}^{s-1} p^{i}\left(\phi_{p^{2 i-2}}+p \phi_{p^{2 i+2}}\right)+\phi_{1}+p \phi_{p^{2}}-p \sum_{i=0}^{s-2} p^{i} \phi_{p^{2 i}} \\
& =\sum_{i=0}^{s} p^{i} \phi_{p^{2 i}} .
\end{aligned}
$$




\section{H. KIM}

As in the proof of part i, write $l=l^{\prime} p^{s}$ with $\left(l^{\prime}, p\right)=1$ and use induction on the number $n(l)$ of prime divisors of $l$. If $n(l)=1$, the assertion is clear. If $n(l)$ is greater than 1 , then

$$
\begin{aligned}
\left.\phi_{1}\right|_{T_{l}} & =\left.\phi_{1}\right|_{T_{l^{\prime}} T_{p^{s}}}=\left.\left(\sum_{\nu \mid l^{\prime}} \nu \phi_{\nu^{2}}\right)\right|_{T_{p^{s}}} \text { by induction on } n(l) \\
& =\sum_{\nu \mid l} \nu \phi_{\nu^{2}} \quad \text { by induction on } s \text { and applying the same argument as before. }
\end{aligned}
$$

We claim that for $d=4 N n-r^{2}$,

$$
J_{m}(d)=- \text { coefficient of } q^{n} \zeta^{r} \text { in } \sum_{u \mid m} 2^{s(u, N)} u \phi_{u^{2}} .
$$

Let $p$ be a prime dividing N. By [Koi, Theorem 6.3(2)] (or [Fer96b, Proposition 2.6]), the generalized Hecke operator $T(p)$ satisfies the following composition rule: for $k \geqslant 0$,

$$
T\left(p^{k}\right) \circ T(p)=T\left(p^{k+1}\right)+p I_{p} \circ T\left(p^{k-1}\right)
$$

where $\left.t_{n}\right|_{I_{p}}=t_{n}^{(p)}$ and $t_{n}$ is defined to be 0 if $n$ is not a rational integer. For $l$ coprime to $p$, we obtain

$$
\begin{aligned}
t_{l p^{k+1}} & =\left.t_{l}\right|_{T\left(p^{k+1}\right)}=\left.\left(\left.t_{l}\right|_{T\left(p^{k}\right)}\right)\right|_{T(p)}-\left.p t_{l}^{(p)}\right|_{T\left(p^{k-1}\right)} \\
& =\left.t_{l p^{k}}\right|_{T(p)}-p t_{l p^{k-1}}^{(p)}=t_{l p^{k}}^{(p)}(p \tau)+\left.p t_{l p^{k}}\right|_{U_{p}}-p t_{l p^{k-1}}^{(p)} .
\end{aligned}
$$

Meanwhile, [Koi, Theorem 3.1, Case I] (or [Fer96b, Theorem 3.7, Case 1]) provides the formula

$$
\left.p t_{l p^{k}}\right|_{U_{p}}+t_{l p^{k}}=t_{l p^{k}}^{(p)}+p t_{l p^{k-1}}^{(p)} .
$$

Combining (3) with (4) we come up with $t_{l p^{k+1}}(\tau)=t_{l p^{k}}^{(p)}(p \tau)+t_{l p^{k}}^{(p)}(\tau)-t_{l p^{k}}(\tau)$ and, therefore,

$\sum_{Q \in \mathcal{Q}_{d, N} / \Gamma_{0}(N)^{*}} \frac{1}{w_{Q}} t_{l p^{k+1}}\left(\alpha_{Q}\right)=\left.\sum_{Q \in \mathcal{Q}_{d, N} / \Gamma_{0}(N)^{*}} \frac{1}{w_{Q}}\left(t_{l p^{k}}^{(p)}(p \tau)+t_{l p^{k}}^{(p)}(\tau)\right)\right|_{\tau=\alpha_{Q}}-\sum_{Q \in \mathcal{Q}_{d, N} / \Gamma_{0}(N)^{*}} \frac{1}{w_{Q}} t_{l p^{k}}\left(\alpha_{Q}\right)$.

The map which sends $[a, b, c] \in \mathcal{Q}_{d, N}$ to $[a / p, b, c p] \in \mathcal{Q}_{d, N / p}$ induces a bijection between $\mathcal{Q}_{d, N} /$ $\Gamma_{0}(N)^{*}$ and $\mathcal{Q}_{d, N / p} / \Gamma_{0}(N / p)^{*}$, and the natural map from $\mathcal{Q}_{d, N} / \Gamma_{0}(N)^{*}$ to $\mathcal{Q}_{d, N / p} / \Gamma_{0}(N / p)^{*}$ also gives a bijection. Thus, (5) is rewritten as

$$
\sum_{Q \in \mathcal{Q}_{d, N} / \Gamma_{0}(N)^{*}} \frac{1}{w_{Q}} t_{l p^{k+1}}\left(\alpha_{Q}\right)=2 \sum_{Q \in \mathcal{Q}_{d, N / p} / \Gamma_{0}(N / p)^{*}} \frac{1}{w_{Q}} t_{l p^{k}}^{(p)}\left(\alpha_{Q}\right)-\sum_{Q \in \mathcal{Q}_{d, N} / \Gamma_{0}(N)^{*}} \frac{1}{w_{Q}} t_{l p^{k}}\left(\alpha_{Q}\right),
$$

which yields

$$
J_{l p^{k+1}}(d)=2 J_{l p^{k}}^{(p)}(d)-J_{l p^{k}}(d) \text { for } k \geqslant 0 .
$$

We divide $N$ into two cases.

Case I: $N=p=2$ or 3 or 5 . In (2) we write $m=l p^{k}$ with $(l, p)=1$. We use induction on $k$ to prove the claim. If $k=0$, the claim (2) follows from Lemma 3.2. Now assume the claim for $k$. We have

$$
\begin{aligned}
J_{l p^{k+1}}(d) & =2 J_{l p^{k}}^{(p)}(d)-J_{l p^{k}}(d) \\
& =- \text { coefficient of } q^{n} \zeta^{r} \text { in }\left[\left.2\left(\left.\phi_{1}^{(p)}\right|_{T_{l p^{k}}}\right)\right|_{V_{p}}-\left(\sum_{i=1}^{k} \sum_{\nu \mid l} 2 \nu p^{i} \phi_{\nu^{2} p^{2 i}}+\sum_{\nu \mid l} \nu \phi_{\nu^{2}}\right)\right]
\end{aligned}
$$




\section{Borcherds PRODUCTS ASSOCIATED WITH CERTAIN THOMPSON SERIES}

by [Zag00, Theorem 5(ii)] and induction hypothesis

$=-$ coefficient of $q^{n} \zeta^{r}$ in $\left[\left.2\left(\sum_{i=0}^{k} \sum_{\nu \mid l} \nu p^{i} \phi_{\nu^{2} p^{2 i}}^{(p)}\right)\right|_{V_{p}}-\left(\sum_{i=1}^{k} \sum_{\nu \mid l} 2 \nu p^{i} \phi_{\nu^{2} p^{2 i}}+\sum_{\nu \mid l} \nu \phi_{\nu^{2}}\right)\right]$

by [Zag00, (19) and Theorem 5(iii)]

$$
\begin{aligned}
=- \text { coefficient of } q^{n} \zeta^{r} \text { in }[ & 2 \sum_{i=0}^{k} \sum_{\nu \mid l} \nu p^{i}\left(p \phi_{\nu^{2} p^{2 i+2}}+\phi_{\nu^{2} p^{2 i}}\right) \\
& \left.-\left(\sum_{i=1}^{k} \sum_{\nu \mid l} 2 \nu p^{i} \phi_{\nu^{2} p^{2 i}}+\sum_{\nu \mid l} \nu \phi_{\nu^{2}}\right)\right]
\end{aligned}
$$

by Lemma 3.1

$=-$ coefficient of $q^{n} \zeta^{r}$ in $\left[\sum_{i=1}^{k+1} \sum_{\nu \mid l} 2 \nu p^{i} \phi_{\nu^{2} p^{2 i}}+\sum_{\nu \mid l} \nu \phi_{\nu^{2}}\right]$

as desired.

Case II: $N=6$. In (2), we write $m=l 2^{k_{1}} 3^{k_{2}}$ with $(l, 6)=1$ and $k_{1}, k_{2} \geqslant 0$. For simplicity, we put $\alpha(u)=2^{s(u, 2)} u$ and $\beta(u)=2^{s(u, 6)} u$. We use induction on $k_{1}+k_{2}$. If $k_{1}+k_{2}=0$, the claim is immediate from Lemma 3.2. Now assume $k_{1}+k_{2} \geqslant 1$, say $k_{2} \geqslant 1$. Then we have

$$
\begin{aligned}
& J_{l 2^{k_{1} 3^{k_{2}}}}(d) \\
& =2 J_{l 2^{k_{1} 3^{k_{2}-1}}}^{(3)}(d)-J_{l 2^{k_{1}} 3^{k_{2}-1}}(d) \quad \text { by }(6) \\
& =- \text { coefficient of } q^{n} \zeta^{r} \text { in }\left[\left.2 \sum_{i=0}^{k_{1}} \sum_{j=0}^{k_{2}-1} \sum_{\nu \mid l} \alpha\left(\nu 2^{i} 3^{j}\right) \phi_{\left(\nu 2^{i} 3^{j}\right)^{2}}^{(3)}\right|_{V_{3}}-\sum_{i=0}^{k_{1}} \sum_{j=0}^{k_{2}-1} \sum_{\nu \mid l} \beta\left(\nu 2^{i} 3^{j}\right) \phi_{\left(\nu 2^{i} 3^{j}\right)^{2}}\right]
\end{aligned}
$$

by the result in the case $N=2$ and induction hypothesis

$$
\begin{aligned}
= & - \text { coefficient of } q^{n} \zeta^{r} \text { in }\left[2 \sum_{i=0}^{k_{1}} \sum_{j=0}^{k_{2}-1} \sum_{\nu \mid l} \alpha\left(\nu 2^{i} 3^{j}\right)\left(3 \phi_{\left(\nu 2^{i} 3^{j+1}\right)^{2}}+\phi_{\left(\nu 2^{i} 3^{j}\right)^{2}}\right)\right. \\
& \left.-\sum_{i=0}^{k_{1}} \sum_{j=0}^{k_{2}-1} \sum_{\nu \mid l} \beta\left(\nu 2^{i} 3^{j}\right) \phi_{\left(\nu 2^{i} 3^{j}\right)^{2}}\right]
\end{aligned}
$$

by Lemma 3.1

$$
\begin{aligned}
= & - \text { coefficient of } q^{n} \zeta^{r} \text { in }\left[\sum_{i=0}^{k_{1}} \sum_{\nu \mid l} 2 \alpha\left(\nu 2^{i} 3^{k_{2}-1}\right) \cdot 3 \phi_{\left(\nu 2^{i} 3^{k_{2}}\right)^{2}}+\sum_{i=0}^{k_{1}} \sum_{j=1}^{k_{2}-1} \sum_{\nu \mid l}\left[2 \alpha\left(\nu 2^{i} 3^{j-1}\right)\right.\right. \\
& \left.\left.\cdot 3+2 \alpha\left(\nu 2^{i} 3^{j}\right)-\beta\left(\nu 2^{i} 3^{j}\right)\right] \phi_{\left(\nu 2^{i} 3^{j}\right)^{2}}+\sum_{i=0}^{k_{1}} \sum_{\nu \mid l}\left[2 \alpha\left(\nu 2^{i}\right)-\beta\left(\nu 2^{i}\right)\right] \phi_{\left(\nu 2^{i}\right)^{2}}\right] \\
= & - \text { coefficient of } q^{n} \zeta^{r} \text { in } \sum_{i=0}^{k_{1}} \sum_{j=0}^{k_{2}} \sum_{\nu \mid l} \beta\left(\nu 2^{i} 3^{j}\right) \phi_{\left(\nu 2^{i} 3^{j}\right)^{2},}
\end{aligned}
$$

as desired.

Let $z \in \mathfrak{H}$. Note that $(1 / m) t_{m}(z)$ can be viewed as the coefficient of $q^{m}$-term in $-\log q-\log (t(\tau)-$ $t(z))$ (see [Nor84]). Thus $\log q^{-1}-\sum_{m>0}(1 / m) t_{m}(z) q^{m}=\log (t(\tau)-t(z))$. Taking exponential on 


\section{H. KIM}

both sides, we get

$$
q^{-1} \exp \left(-\sum_{m>0} \frac{1}{m} t_{m}(z) q^{m}\right)=t(\tau)-t(z) .
$$

Define $B^{*}\left(u^{2}, d\right)=2^{s(u, N)} B\left(u^{2}, d\right)$. By the claim (2), we obtain

$$
J_{m}(d)=-\sum_{u \mid m} u B^{*}\left(u^{2}, d\right) .
$$

From (7) and (8), it follows that

$$
\begin{aligned}
\mathcal{H}_{d}(t(\tau)) & =q^{-H(d)} \exp \left(-\sum_{m=1}^{\infty} J_{m}(d) q^{m} / m\right)=q^{-H(d)} \exp \left(\sum_{m=1}^{\infty} \sum_{u \mid m} u B^{*}\left(u^{2}, d\right) q^{m} / m\right) \\
& =q^{-H(d)} \exp \left(\sum_{m=1}^{\infty} \sum_{u=1}^{\infty} u B^{*}\left(u^{2}, d\right) q^{m u} /(m u)\right) \\
& =q^{-H(d)} \exp \left(\sum_{u=1}^{\infty}\left(-B^{*}\left(u^{2}, d\right)\right) \sum_{m=1}^{\infty}-\left(q^{u}\right)^{m} / m\right) \\
& =q^{-H(d)} \exp \left(\sum_{u=1}^{\infty} \log \left(1-q^{u}\right)^{-B^{*}\left(u^{2}, d\right)}\right)=q^{-H(d)} \prod_{u=1}^{\infty}\left(1-q^{u}\right)^{-B^{*}\left(u^{2}, d\right)} .
\end{aligned}
$$

Now, the fact $A(D, d)=-B(D, d)$ completes the proof of our theorem.

Remark 3.3. If $N=4$, our proof does not apply since in this case the 2-plicate $t^{(2)}$ of $t$ is the Hauptmodul for $\Gamma_{0}(2)$, which is not $\Gamma_{0}(N)^{*}$-invariant for any $N$. In fact, we can numerically check that Theorem 1.1 fails when $N=4$.

\section{Some recursion formulas}

Let $\delta$ be the denominator of $H(d)$. In the course of proving Theorem 1.1 we have seen that

$$
\mathcal{H}_{d}(t(\tau))=q^{-H(d)} \prod_{m=1}^{\infty} \exp \left(-\sum_{u \mid m} u A^{*}\left(u^{2}, d\right) q^{m} / m\right) .
$$

Observe that $\left(q^{H(d)} \mathcal{H}_{d}(t(\tau))\right)^{\delta}$ is of the form $1+\sum_{m=1}^{\infty} c(m) q^{m}$ with $c(m) \in \mathbb{Z}$. Then

$$
1+\sum_{m=1}^{\infty} c(m) q^{m}=\prod_{m=1}^{\infty} \exp \left(-\sum_{u \mid m} \delta u A^{*}\left(u^{2}, d\right) q^{m} / m\right) .
$$

Put $V=\prod_{m=1}^{\infty} \exp \left(-\sum_{u \mid m} \delta u A^{*}\left(u^{2}, d\right) q^{m} / m\right)$. The differential identity $(\log V)^{\prime}=V^{\prime} / V$ (here ${ }^{\prime}$ denotes $q(d / d q)=(1 / 2 \pi i)(d / d \tau))$ leads to

$$
\left(-\sum_{m=1}^{\infty} \sum_{u \mid m} \delta u A^{*}\left(u^{2}, d\right) q^{m}\right) \cdot\left(1+\sum_{m=1}^{\infty} c(m) q^{m}\right)=\sum_{m=1}^{\infty} m c(m) q^{m}
$$

Comparing the coefficients of $q^{m}$ on both sides we get

$$
\sum_{u \mid m} \delta u A^{*}\left(u^{2}, d\right)+\sum_{1 \leqslant k<m} c(m-k)\left(\sum_{u \mid k} \delta u A^{*}\left(u^{2}, d\right)\right)=-m c(m) .
$$




\section{BORCHERDS PRODUCTS ASSOCIATED WITH CERTAIN THOMPSON SERIES}

Now we come up with the following recursion formula for $A^{*}\left(m^{2}, d\right)$ : for $m \geqslant 1$,

$$
A^{*}\left(m^{2}, d\right)=-\frac{1}{\delta} c(m)-\frac{1}{m}\left[\sum_{\substack{1 \leqslant u<m \\ u \mid m}} u A^{*}\left(u^{2}, d\right)+\sum_{1 \leqslant k<m} c(m-k)\left(\sum_{u \mid k} u A^{*}\left(u^{2}, d\right)\right)\right] .
$$

Thus all $A^{*}\left(m^{2}, d\right)$ can be computed from the values of $c(m)$. Likewise all $c(m)$ can be estimated recursively from the values of $A^{*}\left(m^{2}, d\right)$.

Example $4.1(N=2, d=4)$. Theorem 1.1 yields the following product formula:

$$
\left(t(\tau)-t\left(\frac{1+\sqrt{-1}}{2}\right)\right)^{1 / 2}=q^{-1 / 2} \prod_{u=1}^{\infty}\left(1-q^{u}\right)^{A^{*}\left(u^{2}, d\right)} .
$$

Here the Hauptmodul $t$ for $\Gamma_{0}(2)^{*}$ can be described by means of Dedekind $\eta$-functions, i.e.

$$
\begin{aligned}
t(\tau) & =\left(\frac{\eta(\tau)}{\eta(2 \tau)}\right)^{24}+24+4096\left(\frac{\eta(2 \tau)}{\eta(\tau)}\right)^{24} \\
& =q^{-1}+4372 q+96256 q^{2}+1240002 q^{3}+10698752 q^{4}+74428120 q^{5}+\cdots
\end{aligned}
$$

from which we obtain $t((1+\sqrt{-1}) / 2)=-104$. The identity (10) is then rewritten as

$$
1+104 q+4372 q^{2}+\cdots=\prod_{u=1}^{\infty}\left(1-q^{u}\right)^{2 A^{*}\left(u^{2}, d\right)}=\prod_{m=1}^{\infty} \exp \left(-\sum_{u \mid m} 2 u A^{*}\left(u^{2}, d\right) q^{m} / m\right) .
$$

In (9), we take $\delta=2, c(1)=104, c(2)=4372, c(3)=96256$, etc. Then

$$
\begin{aligned}
& A^{*}(1,4)=-\frac{1}{2} c(1)=-52, \\
& A^{*}(4,4)=-\frac{1}{2} c(2)-\frac{1}{2}\left[A^{*}(1,4)+c(1) A^{*}(1,4)\right]=544, \\
& A^{*}(9,4)=-\frac{1}{2} c(3)-\frac{1}{3}\left[A^{*}(1,4)+c(2) A^{*}(1,4)+c(1) A^{*}(1,4)+c(1) \cdot 2 \cdot A^{*}(4,4)\right]=-8244,
\end{aligned}
$$

\section{ACKNOWLEDGEMENTS}

I am grateful to Professor Don Zagier for introducing me to this subject. I would also like to take an opportunity to thank Professor Richard E. Borcherds, Professor Jan H. Bruinier and Professor Ja Kyung Koo for their kind and valuable comments.

\section{Appendix A}

Let $f_{0}=\theta$. We found the initial $f_{d}$ 's by expressing $\left[f_{0}, E_{12-2 n}(4 N \tau)\right]_{n} / \Delta(4 N \tau)$ (if necessary, $\left.\left[f_{d}, E_{12-2 n}(4 N \tau)\right]_{n} / \Delta(4 N \tau)\right)$ as linear combinations of them for $n=1,2,3,4$. Here $E_{k}$ is the normalized Eisenstein series of weight $k, \Delta$ is the modular discriminant and $[\cdot, \cdot]_{n}$ denotes the 'Cohen bracket' (see [Coh75, § 7] or [Zag94, § 1]).

$$
\begin{aligned}
N= & 2 \\
f_{4}= & q^{-4}-52 q+272 q^{4}+2600 q^{8}-8244 q^{9}+15300 q^{12}+71552 q^{16}-204800 q^{17} \\
& +282880 q^{20}+\cdots, \\
f_{7}= & q^{-7}-23 q-2048 q^{4}+45056 q^{8}+252 q^{9}-516096 q^{12}+4145152 q^{16}-1771 q^{17} \\
& -26378240 q^{20}+\cdots,
\end{aligned}
$$




\section{H. KIM}

$$
\begin{aligned}
N & =3 \\
f_{3} & =q^{-3}-14 q+40 q^{4}-78 q^{9}+168 q^{12}-378 q^{13}+688 q^{16}+\cdots \\
f_{8} & =q^{-8}-34 q-188 q^{4}+2430 q^{9}+8262 q^{12}-11968 q^{13}-34936 q^{16}+\cdots \\
f_{11} & =q^{-11}+22 q-552 q^{4}-11178 q^{9}+48600 q^{12}+76175 q^{13}-269744 q^{16}+\cdots \\
N & =5 \\
f_{4} & =q^{-4}-8 q+q^{4}+10 q^{5}+12 q^{9}-62 q^{16}+65 q^{20}+\cdots \\
f_{11} & =q^{-11}-12 q-56 q^{4}-45 q^{5}+276 q^{9}+672 q^{16}+2520 q^{20}+\cdots \\
f_{15} & =q^{-15}-38 q+112 q^{4}-96 q^{5}-988 q^{9}+8512 q^{16}+11856 q^{20}+\cdots \\
f_{16} & =q^{-16}-6 q-132 q^{4}+120 q^{5}-1014 q^{9}+3585 q^{16}+17030 q^{20}+\cdots \\
f_{19} & =q^{-19}+20 q+56 q^{4}-210 q^{5}-780 q^{9}-23200 q^{16}+46760 q^{20}+\cdots \\
N & =6 \\
f_{8} & =q^{-8}-10 q-12 q^{4}+54 q^{9}+54 q^{12}-88 q^{16}+\cdots \\
f_{12} & =q^{-12}-28 q+26 q^{4}-156 q^{9}+168 q^{12}+728 q^{16}+\cdots \\
f_{15} & =q^{-15}-10 q-64 q^{4}+3 q^{9}-320 q^{12}+1664 q^{16}+\cdots \\
f_{20} & =q^{-20}+12 q-64 q^{4}-756 q^{9}+945 q^{12}-2912 q^{16}+\cdots \\
f_{23} & =q^{-23}-13 q+64 q^{4}-27 q^{9}-1728 q^{12}-5760 q^{16}+\cdots
\end{aligned}
$$

For the remaining $f_{d}(\tau)$, we inductively obtain them by multiplying $f_{d-4 N}(\tau)$ by $j(4 N \tau)$ to get a 'plus' form of weight $\frac{1}{2}$ with leading coefficient $q^{-d}$, and then subtracting a suitable linear combination of $f_{d^{\prime}}(\tau)$ with $0 \leqslant d^{\prime}<d$.

\section{REFERENCES}

ACMS92 D. Alexander, C. Cummins, J. Mckay and C. Simons, Completely replicable functions, in Groups, combinatorics and geometry (Cambridge University Press, 1992), 87-95.

Bor92 R. E. Borcherds, Monstrous moonshine and monstrous Lie superalgebras, Invent. Math. 109 (1992), 405-444.

Bor95 R. Borcherds, Automorphic forms on $O_{s+2,2}(\mathbb{R})$ and infinite products, Invent. Math. 120 (1995), 161-213.

Bor98 R. Borcherds, Automorphic forms with singularities on Grassmannians, Invent. Math. 132 (1998), 491-562.

Bru02 J. H. Bruinier, Borcherds products on $O(2, l)$ and Chern classes of Heegner Divisors, Habilitationsschrift (Universität Heidelberg, May 2000), Lecture Notes in Mathematics, vol. 1780 (SpringerVerlag, Berlin, 2002).

Coh75 H. Cohen, Sums involving the values at negative integers of L-functions of quadratic characters, Math. Ann. 217 (1975), 271-285.

EZ85 M. Eichler and D. Zagier, The theory of Jacobi forms, Progress in Mathematics, vol. 55 (Birkhäuser, Basel, 1985).

Fer96a C. R. Ferenbaugh, Lattices and generalized Hecke operators, in Groups, difference sets and the Monster, eds K. T. Arasu et al. (de Gruyter, Berlin, 1996), 363-368.

Fer96b C. R. Ferenbaugh, Replication formulae for $n \mid$ h-type Hauptmoduls, J. Algebra 179 (1996), 808-837.

FLM84 I. B. Frenkel, J. Lepowsky, A. Meurman, A natural representation of the Fischer-Griess Monster with the modular function $J$ as character, Proc. Natl Acad. Sci. USA 81 (1984), 3256-3260.

FLM88 I. B. Frenkel, J. Lepowsky and A. Meurman, Vertex operator algebras and the Monster (Academic Press, Boston, MA, 1988). 


\section{BORCHERDS PRODUCTS ASSOCIATED WITH CERTAIN THOMPSON SERIES}

KKKO S. J. Kang, C. H. Kim, J. K. Koo and Y. T. Oh, Graded Lie superalgebras and super-replicable functions, Preprint.

Koi M. Koike, On replication formula and Hecke operators, Nagoya University, Preprint.

Nor84 S. P. Norton, More on moonshine, in Computational group theory (Academic Press, 1984), 185-193.

Zag94 D. Zagier, Modular forms and differential operators, Proc. Indian Acad. Sci. 104 (1994), 57-75.

Zag00 D. Zagier, Traces of singular moduli, Max-Planck-Institut für Mathematik, Preprint (2000).

Chang Heon Kim chkim@swu.ac.kr

Department of Mathematics, Seoul Women's University, 126 Kongnung 2 dong, Nowon-gu, Seoul, 139-144 South Korea 